\title{
POLARIZED LIGHT STUDIES OF CORNEAL STRUCTURE*
}

\author{
BY \\ E. J. NAYLOR \\ Department of Ophthalmology, University of Manchester
}

THE importance of the cornea as the major refracting surface of the eye and the peculiar circumstance of its transparency have given rise to investigations since early times. Recent work on the hydration properties of the cornea, for example that of Cogan and Kinsey (1942) and Hart and Chandler (1948), seems to indicate that the problem of transparency will be solved only by an attack over a wide field. It is also apparent from the literature that our knowledge of the fine corneal structure is still limited, and that comparatively little has been done to apply techniques other than that of visual microscopy. These include the use of the electron microscope, $x$-ray diffraction, and polarized light, all of which have been applied with success to the elucidation of the structure of other tissues. The literature contains reports of investigations of eye tissues by electron microscopy (Sebruyns, 1950) and of $x$-ray diffraction (Hertel, 1933), and a paper describing the use of an ultra-violet absorption technique for investigating corneal transparency (Caspersson and Engström, 1946). The relevant literature on polarized light is quoted by Cogan (1941), and has been briefly reviewed by Stanworth and Naylor (1950).

In the latter paper the phenomenon of the interference figure was discussed in terms of photo-elasticity and crystal optics. The qualitative results of this investigation together with some additional observations made by the present author have been further analysed (Stanworth and Naylor, 1952), and it has been shown that the coefficient of birefringence of the cornea is 0.0014. Considered as a lamina made up of a series of anisotropic fibres arranged randomly in layers parallel to the surface, the coefficient of birefringence of the constituent fibres, according to theoretical calculations, should be about $0 \cdot 0028$, i.e., twice that of the lamina.

The term "birefringence" is often used in different ways, and various adjectives have been employed by different authors to connote specific meanings. In its widest sense it is synonymous with double-refraction or optical anisotropy in indicating that light travels at different speeds-and that, therefore, different refractive indices are found for the substanceaccording to the direction of travel and the direction of vibration of the incident light. When used quantitatively, " effective birefringence " indicates

* Received for publication October 3, 1952. 
the difference between the ordinary and the extraordinary refractive indices for any direction of light path, and " coefficient of birefringence" indicates the maximum value of the birefringence, which occurs when the light is vibrating along the principal axes of the substance. A quantitative value for " birefringence" in what follows-and usually in the literature-implies the coefficient of birefringence.

It is the purpose of this paper to report some of the findings of an investigation originally designed to verify the above analysis experimentally, and later extended to include the application of techniques involving polarized light to studies of the submicroscopic structure of the corneal stroma. Details of the methods are published elsewhere (Naylor, 1952).

\section{Microscopic Structure}

Attempts to isolate a piece of corneal stroma in the form of a single fibre or lamella failed, and histological sections were therefore made by freezing or fixing techniques. Examination of these sections, typical photographs of which are seen in Fig. 1, confirm the findings of His (1856) that the corneal fibres are birefringent with optic (slow) axes along their length. In anteroposterior sections of the cornea, some fibre layers will be encountered in which the fibres have been cut perpendicular to their length, along their length, or in intermediate positions. The first-named will show no effective
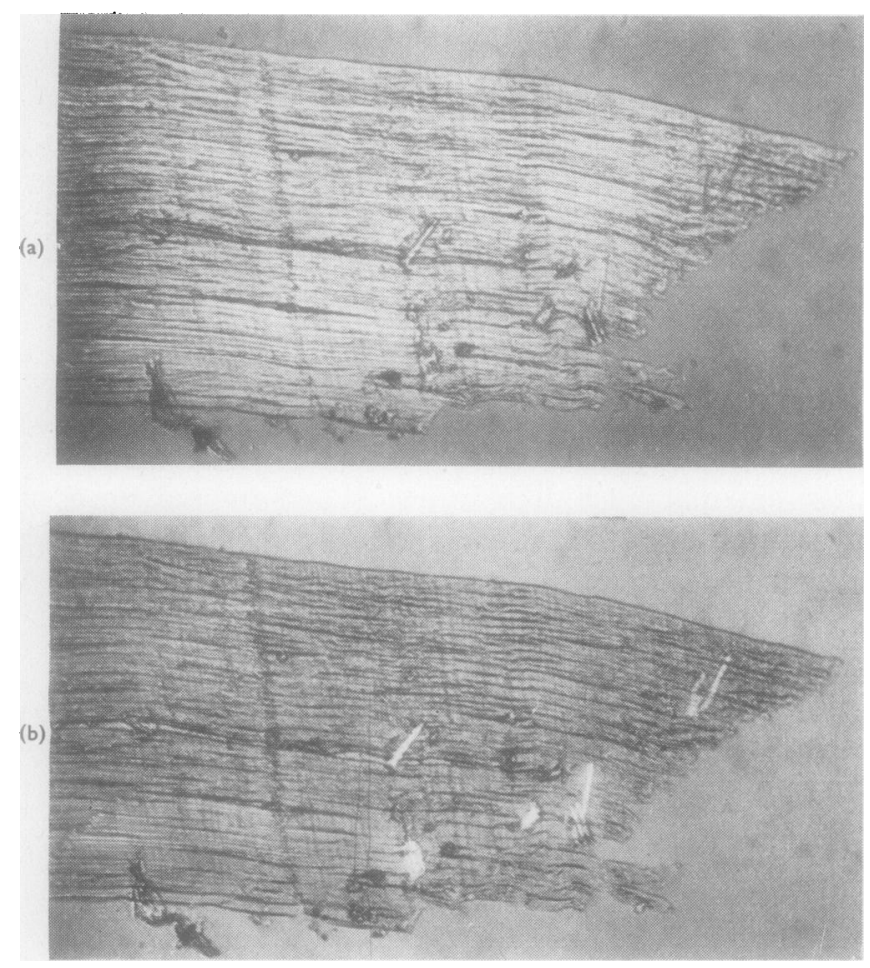

FIG. 1.-Antero-posterior section of cat cornea, $25 \mu$ thick, photographed under polarizing microscope with crossed polars and sensitive tint plate. $\times 80$.

Specimen in diagonal position with slow axis of tint plate: (a) perpendicular to fibre direction, fibres appearing orange; (b) parallel to fibre direction, fibres appearing blue. 
birefringence, since the light will be travelling parallel to their optic axis; the second type will show the maximum effect; the last-named will produce effects of varying magnitude according to their inclination to the plane of the section. It was thought that the course of the corneal fibres might thus be mapped out from readings of the retardation they produced, but the technique of measurement is not sufficiently delicate to enable this to be done with certainty. Nevertheless, large numbers of readings were taken on many sections cut in various ways and a statistical plot of these results showed no support for the suggestion put forward by Kokott (1938) of preferential orientation of fibres in layers at different depths in the cornea. It is not possible directly to measure the coefficient of birefringence $(\omega)$ but one can assess with accuracy the relative retardation $(\Delta)$ of the light components issuing from the doubly refractile corneal elements and it can be shown from first principles that $\Delta=d$. $\omega$. $\sin \alpha \cdot \tan \alpha$, where $\alpha$ is the angle that the direction of passage of the light makes with the optic axis and $d$ is the thickness of the section.

However, the coefficient of birefringence of the fibres was obtained from the maximum readings of the retardation, by dividing the latter by the thickness of the section. The most constant results were obtained with sections 15-25 $\mu$ thick, mounted in Ringer-Locke solution, and these gave a figure of 0.0030 for the coefficient of birefringence. This value is in accordance with the prediction of 0.0028 .

Another interesting observation is that with the aid of polarized light about one hundred distinct zones can be counted in the cat cornea, compared with forty visible under ordinary illumination. This is not readily demonstrable in an illustration based on a single photograph, for what appears in the latter as a single zone of the same intensity may be resolved visually into two or more zones by a slight rotation of the polarizer or analyser. The human cornea is usually stated to contain about 60 lamellae; this figure was given by Bowman (1849) who, however, pointed out that no exact estimate could be given. It seems probable that this number is too low and that 150 would be a better estimate on this basis.

\section{SUb-Microscopic Structure}

The standard biological technique employed as a preliminary to polarization analysis of tissue structure is to immerse the object consecutively in fluids of different refractive indices and then measure the retardation. These values are then plotted with refractive indices as abscissae and coefficients of birefringence as ordinates to give a curve similar to that derived from the well-known Wiener relationship (see, for example, Schmitt, 1939). Such a curve indicates whether the tissue exhibits intrinsic birefringence due to molecular asymmetry, or form birefringence due to the regular arrangement of asymmetrical aggregates of molecules (micelles), or both; it also reveals the probable nature of the micelles and their refractive index. The standard 
technique was not directly applicable to the cornea because of its isotropic appearance when viewed normally, and also because it was not possible to isolate individual fibres satisfactorily. A method was devised, however (Naylor, 1952) by which the retardation was measured for light passing obliquely through circular disks of cornea removed by means of a trephine punch and the birefringence of the constituent fibres computed therefrom. Over one hundred disks were examined from cat corneae from freshly enucleated eyes, and from eyes preserved in either $2 \frac{1}{2}$ per cent. formal saline, 5 per cent. mercuric chloride, or 35 per cent. alcohol. These corneal disks exhibited both form and intrinsic birefringence of positive sign, the values of the latter for the above categories being $0.000450,0.000525,0.000388$, and 0.000250 respectively. The refractive index of the micelles was found to be between 1.50 and 1.52 , which is low compared to that of most other physiological proteins, and calculations from the Wiener formula suggest that they occupy somewhat less than 3 per cent. of the total volume of the corneal substance. The presence of intrinsic birefringence denotes molecular asymmetry, and its positive sign indicates that the molecules have their long axes in the fibre direction. Form birefringence is due to the asymmetry of the molecular aggregates or micelles, and the finding that it has a positive sign indicates that the corneal micelles are of the rodlet type.

Intrinsic Birefringence.-In preparations which differ only in their degree of swelling, assuming no change in the orientation or in the birefringence of the molecule itself, the intrinsic birefringence should be inversely proportional to the volume. In the cornea practically all the swelling occurs at right angles to the plane of the fibres, and therefore the products of intrinsic birefringence and the thickness of the disks should be approximately constant. The mean figures for 27 disks fixed in formalin, fourteen disks fixed in mercury, and fifteen disks fixed in alcohol are $2.68 \times 10^{-4}, 2.33 \times 10^{-4}$, and $2.60 \times 10^{-4}$, for thicknesses of 0.52 , 0.60 , and $1.06 \mathrm{~mm}$. respectively: it may be deduced, therefore, that swelling and fixation bring about no gross disorientation of the molecules. It is of interest to observe the values obtained for disks of different thicknesses, all fixed by the same method, but the range of thickness in the present experiments was not sufficient to allow this to be done except in the case of the fifteen alcohol-fixed disks, which varied in thickness from 0.76 to $1.33 \mathrm{~mm}$. Divided into one group of three disks all with mean thickness $0.83 \mathrm{~mm}$., and three groups of four disks with mean thickness $0.95 \mathrm{~mm} ., 1.12 \mathrm{~mm}$., and $1.27 \mathrm{~mm}$. respectively, the corresponding figures for the products of intrinsic birefringence and thickness are $2.80 \times 10^{-4}$, $2 \cdot 56 \times 10^{-4}, 2 \cdot 72 \times 10^{-4}$, and $2 \cdot 22 \times 10^{-4}$. This does not suggest undue disorientation of the molecules, although the number of readings available is hardly sufficient to enable one to be dogmatic. (Correlation coefficient, $r$, between $\omega$ and $d=-0 \cdot 73$; probability integral, $P,=0 \cdot 25$ ).

Form Birefringence.-Although the protein content of the cat cornea is about 25 per cent. (Duane, 1949), the calculated volume of the micelles from the present results is less than 3 per cent. A low value is not unexpected in such a highly aqueous structure as the cornea. Possible explanations, not mutually exclusive, are: 
(i) that the major portion of the corneal protein may be in unaggregated molecular chains;

(ii) that the molecules may be aggregated into fringed micelles having boundaries less well defined than those in the ideal mixed body considered by Wiener;

(iii) that the micelles, though sharply defined, may not lie parallel to the long axis of the fibres.

Some support for the last supposition is offered by the electron microscope studies of Sebruyns (1950) who found a helicoidal arrangement of fibrils. The last two considerations lead to the deduction that 3 per cent. represents too low a value for the true micellar volume, and the electron microscope photographs by Sebruyns (1950), showing tracts inclined at angles of about $45^{\circ}$, indicate that the extent of the underestimate is not inconsiderable. The figure of 5 per cent. would probably be a better estimate as it yields a value of 0.008 for the micellar intrinsic birefringence (intrinsic birefringence divided by micellar volume). This accords well with that obtained for other protein micelles; for example, Bear, Schmitt, and Young (1937) found 0.005 for nerve axoplasm, and Weber (1934) found 0.008 for myosin.

The low value of the micellar volume could be due either to a large number of very small particles or to a smaller number of larger particles. These two types, of course, are both small with respect to the wavelength of light, and the polarization technique cannot distinguish between them. On the basis of $x$-ray diffraction studies, Hertel (1933) estimated the micellar size as about $1 \mathrm{~m} \mu \times 10 \mathrm{~m} \mu$; there would thus be about $5 \times 10^{15}$ per cubic millimeter of cornea.

If the micelles remained unchanged during swelling of the cornea, the experimental results might be expected to show that the product of micellar volume (expressed as a fraction of the total volume) and thickness remained constant. The respective mean values for disks fixed in formalin, mercury, and alcohol, however, are $0.0156,0 \cdot 0180$, and 0.0239 , for mean thicknesses of $0.52 \mathrm{~mm}$., $0.60 \mathrm{~mm}$., and $1.06 \mathrm{~mm}$., which suggests that the size of the micelle increases during swelling. The swelling of the cornea, then, is at least partially intra-micellar, a finding which agrees with $x$-ray diffraction observations on collagen (Küntzel and Prakke, 1933), and on the cornea (Hertel, 1933). The above figures indicate that for an increase of 100 per cent. in thickness the micelles swell by about 50 per cent. in the fixed cornea, a figure which may be greatly increased in fresh material or in the living state. If the absorbed fluid had its normal refractive index of about $1 \cdot 34$, it might be expected that such a swelling would result in a fall in the refractive index of the micelles to about 1.45 (according to the law of Gladstone and Dale, 1863). Such an effect was not observed. There is, however, no evidence that the formula of Gladstone and Dale applies to intra-micellar changes. Indeed, these authors quote experiments in which the formula failed to apply when hydrates were formed, and it would be surprising if no such effects occurred in the submicroscopic spaces involved in these changes. In addition, the concept of a hydration layer, which will be put forward later, involves the assumption that in such circumstances the fluid has a higher density and hence a higher refractive index than $1 \cdot 34$, and consequently that the value of the micellar refractive index to be expected is not calculable.

Schema of Corneal Structure

It may be helpful, in conclusion, to put forward a schema of the sub- 
microscopic and microscopic anatomy of the corneal stroma in order to introduce a terminology for its constituent parts; if generally adopted this would avoid the uncertainty which often exists at present. The cornea consists chiefly of collagen, the molecules of which, like those elsewhere in the body, consist of long chains, running, in general, parallel to the length of the fibrils which are about $0 \cdot 3-0.6 \mu$ in diameter (Caspersson and Engström, 1946). Within the fibril some of the molecules are aggregated together to form micelles (Fig. 2), there being a continuous range in size of the water-holding spaces between the molecules in the intra-micellar regions

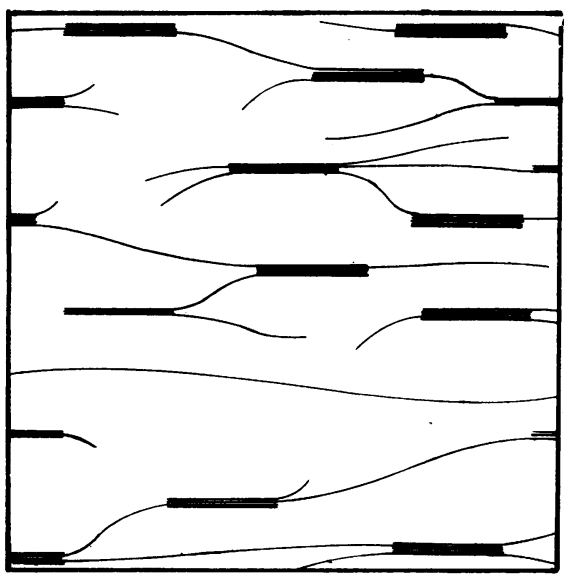

FIG. 2.-Diagram of interior of corneal fibril showing long chain molecules and micelles. ranging from about $1 \mathrm{~m} \mu$ upwards (Frey-Wyssling, 1948). The fibrils themselves are aggregated together to form fibres which run parallel to their neighbours at the same depth in the cornea, but which cross at an angle to those more superficial or more deep thus forming sheets parallel to the surface. It is proposed that the term lamellae be reserved for these sheets of fibres of which there are about one hun dred in the cat cornea, each about $5 \mu$ thick. The spaces between the fibrils in any one fibre, and even between the fibres in any one lamellae, may be merely large spaces of the same type as are found in the interior of the fibril. This would account for the markedly homogeneous nature of the lamellae in corneal sections examined by ordinary microscopy. The spaces between any two lamellae, however, are probably of a fundamentally different nature. The fibres in different lamellae will arise from different cells in the process of development, whilst those in any individual lamella may often arise by a process of division from the same cell. This accounts for the relative ease with which the cornea may be split along the lamellar boundaries in corneal surgery. The directions in which the fibres run in successive lamellae are such as to form a random distribution for the cornea as a whole. It is as if the embryonic cornea was formed originally from a few random cells at different depths, which, by a process of lateral division presumably conditioned by the rising intra-ocular pressure, give rise to the sheets of fibres we now distinguish as lamellae.

In order to consider the relationship of the structure to the property of transparency, it is essential to know the refractive indices of the various components. That of the micelles has been found to be 1.50-1.52; it must 
be pointed out again, however, that this figure is based on a formula which postulates small particles with sharp boundaries, whereas micelles of the fringed type will have more gradual boundaries. It is probable, then, that this figure underestimates the refractive index in the centre of the micelle. In estimating the amount of scatter of light from such a particle no formula is available, the only comparable one being that of Rayleigh for isotropic spherical particles, conditions which do not obtain here. Nevertheless, it seems probable, that, as in Rayleigh's formula, a larger particle, within the limits of size under consideration, would tend to give more scattering. The high transparency, therefore, indicates that the micelles are extremely small.

The second boundary at which light loss might take place is that of the fibril. In discussing the light loss at this boundary, Caspersson and Engström (1946) have taken as a basis for calculations a refractive index of 1.385 for the fibril and one of 1.355 for the surrounding fluid; they have shown that if the transition were abrupt the substance should be completely opaque. They therefore postulate that the transition of refractive index is gradual. It should be pointed out that the figure of 1.385 for the refractive index of the fibril is a doubtful one. All methods of direct measurement of the refractive index of the corneal substance, whether on pieces of cornea or on corneal sections, must involve much larger elements than the fibrils. In structures of this size, the resultant value will be approximately a combination of the refractive index of the fibril and that of the inter-fibrillar fluid according to the admixture formula of Gladstone and Dale (1863), checked for protein solutions by Adair and Robinson (1930). Since the refractive index of the fibril will be underestimated, the argument of Caspersson and Engström still stands. It seems possible that the solution to the problem of the possible cause of this gradual transition lies in the fact that the fibril is of a size just sufficient to involve the formation of phase boundaries (FreyWyssling, 1948). In this case one might expect a hydration layer of increasing density of water molecules as the core of the fibril is approached (Fig. 3), and the refractive index at the edge of the fibril may then have a gradually increasing value towards the core. Some such arrangement might be expected to occur at the fibre boundary.

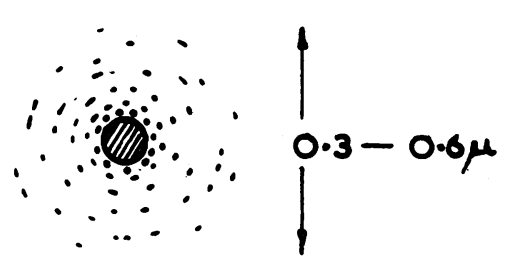

FIG. 3.-Diagram of molecular hydration layer associated with corneal fibril.

It is of some interest and importance to consider how fixatives may alter the transparency. Since the results from these studies in different fixatives can be largely explained on the basis of alterations in degrees of swelling, and the birefringence of the cornea is unaffected by fixation, it appears that the fixatives do not greatly disturb either the molecular chains or the micelles. The opacity they usually produce must, therefore, be related primarily to 
the phase boundaries. This is not surprising, particularly in the case of alcohol which is a markedly hydrophilic and surface-acting substance. It is possible that alcohol disturbs the hydration layer and causes the formation of a sharp boundary, a process analogous to coacervation. The conditions are then, as Caspersson and Engström have proved, such as to cause much light scatter. The fact that the alcohol-fixed cornea becomes maximally transparent when immersed in fluids of refractive index 1.54-1.58 (Aurell and Holmgren, 1946) indicates that the resultant alcohol-fixed fibril has a refractive index of this value. It does not, of course, indicate that this is its value in the normal state.

\section{Summary}

The corneal stroma of the cat has been investigated by polarized light techniques adapted for the purpose. It is suggested that a small proportion (say, 5 per cent.) of the long-chain molecules form extremely small micelles, the nature and distribution of which are discussed; these have the (comparatively) low refractive index of $1 \cdot 50-1 \cdot 52$. These aggregated and unaggregated molecules make up the corneal fibrils, around which lie hydration layers of varying density; bundles of these fibrils constitute the corneal fibres which form about one hundred layers (lamellae) each about $5 \mu$ thick. No evidence has been found of any preferential orientation of fibre directions in different lamellae. The origins of the interfibrillar and interlamellar spaces are considered and the effects of swelling and the relationship of the structure to transparency in the fresh and fixed cornea are discussed.

\section{REFERENCES}

AdaIR, G. S., and Robinson, M. E. (1930). Biochem. J., 24, 993.

Aurell, G., and Holmgren, H. (1946). Nord. Med., 30, 1277.

Bear, R. S., SChmitt, F. O., and Young, J. Z. (1937). Proc. roy. Soc. B., 123, 505.

Bowman, W. (1849). "Lectures on the Parts Concerned in the Operations on the Eye, and on the Structure of the Retina ". Longman, Brown, Green, and Longmans.

Caspersson, T., and Engström, A. (1946). Nord. Med., 30, 1279.

Cogan, D. G. (1941). Arch. Ophthal., Chicago, 25, 391. and KINSEY, V. E. (1942). Ibid., 28, 661.

DuAne, T. D. (1949). Amer. J. Ophthal., 32, No. 6, part 2. 203.

FreY-Wyssling, A. (1948). "Submicroscopic Morphology of Protoplasm and its Derivatives", trans. J. J. Hermans and M. Hollander. Elsevier, New York.

Gladstone, J. H., and Dalé, T. P. (1863). Philos. Trans., 153, 317.

HART, W. M., and Chandler, B. F. (1948). Arch. Ophthal., Chicago, 40, 601.

Hertel, E. (1933). Arch. Augenheilk., 107, 259.

His, W. (1856). "Beiträge zur normalen und pathologischen Histologie der Cornea". Schweighauser, Basel.

Koкотт, W. (1938). v. Graefes Arch. Ophthal., 138, 424.

Küntzel, A., and PrakKe, F. (1933). Biochem. Z., 267, 243. Quoted by Frey-Wyssling, p. 205.

NAYLOR, E. J. (1952). Quart. J. micr. Sci. In the press.

Schmitt, F. O. (1939). Physiol. Rev., 19, 270.

SebruYns, M. (1950). Ann. Oculist., Paris, 183, 483.

StANWORTH, A., and NAYLOR, E. J. (1950). British Journal of Ophthalmology, 34, 282.

(1952). J. exp. Biol. In the press.

Weber, H. H. (1934). Pflügers Arch. ges. Physiol., 235, 205. 